\title{
Carcinoid Heart Disease: Early Outcomes after Surgical Valve Replacement in Nine Patients
}

\author{
Syed Saleem Mujtaba, FRCS, FRCS CTh, Stephen Clark, FRCS, FRCS CTh, PhD \\ Department of Cardiothoracic Surgery, Freeman Hospital, Newcastle upon Tyne, United Kingdom
}

\section{ABSTRACT}

Aim: To describe the early outcomes of carcinoid patients undergoing surgical heart valve replacement.

Methods: In a retrospective study, records of patients with symptomatic carcinoid heart disease referred for valve surgery between 2012 and 2016 were reviewed. The perioperative and early postoperative outcomes were analyzed.

Results: Nine patients, with a mean age of 61 years (range 55-70 years) underwent cardiac surgery for carcinoid syndrome. 3 patients had quadruple valve replacement, 5 had tricuspid and pulmonary valves changed, while one had tricuspid, pulmonary, and aortic valves replaced. Right-sided valves were replaced with biological valves in 8 patients and a mechanical valve in 1 patient. Left-sided valves were replaced with a mechanical valve in 2 patients and with a biological valve in 1 patient. Mean postoperative follow-up was 24 months (range 6-50 months, median 16 months). All patients had a good left ventricle except one, in whom it was mildly impaired. The right ventricle was severely dilated in 4 patients, moderately in 2 , and mildly in 3 . One patient died of heart failure 10 days postoperatively. Functional improvement was noted in all survivors, and they were in New York Heart Association class I at last follow up.

Conclusion: Although carcinoid syndrome is a rare and progressive disease, valve replacement in symptomatic patients is a reasonable option with survival benefit, low early postoperative mortality, without valve-related complications, and with functional improvement. Cardiac assessment is required in all patients with carcinoid disease from the earliest time of medical treatment to improve patients' result.

\section{INTRODUCTION}

Carcinoid is a rare neuroendocrine tumour that occurs in 1.2 to 2.1 persons per 100,000 per year [Connolly 2001a]. The appendix and the terminal ileum are the most common sites for the primary carcinoid tumor [Connolly $2001 \mathrm{~b}]$. The tumor products released in the portal circulation are inactivated by the liver. However, when liver metastases occur, these vasoactive substances can reach

Received fune 15, 2017; received in revised form fuly 30, 2017; accepted August 4, 2017.

Correspondence: Syed Saleem Mujtaba, Department of Cardiothoracic Surgery, Freeman Hospital, Newcastle upon Tyne NE7 7DN, United Kingdom; +44-7404685304 (saleemmujtaba@yaboo.com). the right side of the heart without being inactivated by the first-pass metabolism in the liver, and carcinoid heart disease might evolve [Bernheim 2007; Raja 2010]. At diagnosis, $20 \%$ to $30 \%$ of patients have disseminated disease and consequent carcinoid syndrome [Castillo 2008a], which is characterized by facial flushing, diarrhea, and bronchospasm. Once carcinoid syndrome has developed, approximately $50 \%$ of these patients will go on to develop carcinoid heart disease. The cardiac manifestations are caused by 5-hydroxytryptamine (5-HT or serotonin) released by the malignant cells rather than any direct metastatic involvement of the heart.

Carcinoid heart disease distinctively involves tricuspid and pulmonary valves, and patients often present with right-sided heart failure. Mitral and aortic valve involvement is reported $<10 \%$ of patients with carcinoid heart disease. Preferential right heart involvement is most likely related to inactivation of the vasoactive substances by the lungs. Thus, carcinoid disease of the left-sided heart valves may occur more frequently in patients with an atrial septal defect, patent foramen ovale (PFO), bronchopulmonary carcinoid, or high levels of circulating serotonin [Gustafsson 2008].

Carcinoid heart disease is characterized histologically by diffuse collections of thick, pearly white plaque composed of smooth muscle cells called myofibroblast. [Ferrans 1976]. These plaques are deposited on the endocardial surface of the right atrium and on the endocardial surfaces of the valves on the right side of the heart, resulting in characteristic pathologic and echocardiographic features, which include thickening and immobility of the tricuspid valve and pulmonary valve leaflets [Roberts 1997; Pellikka 1993]. It results in combined stenosis and regurgitation.

Diagnosis is confirmed by elevation of the byproduct of serotonin metabolism, 5-hydroxy indole acetic acid (5-HIAA). The urinary 5-HIAA (24-hour collection) provides a reliable biological marker for the assessment of tumor activity and the response to therapy [Moertel 1987].

The purpose of this study was to present the early outcomes of valve surgery in patients with carcinoid heart disease in our specialized center.

\section{MATERIALS AND METHODS}

Between December 2011 and June 2016, 9 patients underwent valve replacement for carcinoid heart disease at our institution. 3 patients had quadruple valve replacement, 5 had tricuspid and pulmonary valves changed, while one had tricuspid, pulmonary and aortic valves changed. All had been 
Preoperative, Intraoperative, and Postoperative Characteristics of Carcinoid Patients Operated for Valve Replacement

\begin{tabular}{|c|c|c|c|c|c|c|c|c|c|}
\hline Sex & M & $\mathrm{F}$ & $\mathrm{F}$ & $\mathrm{F}$ & $\mathrm{F}$ & M & $\mathrm{F}$ & M & M \\
\hline NYHA, preoperative & 3 & 4 & 3 & 3 & 2 & 3 & 3 & 3 & 3 \\
\hline NYHA, postoperative & 1 & 1 & 1 & 1 & 1 & 1 & 1 & 1 & 1 \\
\hline Surgery date & Aug 2015 & Nov 2015 & Aug 2016 & Dec 2012 & Mar 2013 & Apr 2014 & Feb 2015 & Jan 2016 & Jun 2016 \\
\hline PFO & No & No & No & No & No & large & No & No & No \\
\hline$X$-clamp, $\min$ & 177 & 197 & 164 & 0 & 0 & 82 & 104 & 91 & 85 \\
\hline Bypass, min & 209 & 229 & 195 & 91 & 120 & 142 & 159 & 112 & 96 \\
\hline Aortic regurgitation & M-S & M-S & $S$ & - & - & $S$ & - & - & - \\
\hline Tricus. regurgitation & $S$ & $S$ & MIL & $S$ & $S$ & $S$ & $S$ & $S$ & $S$ \\
\hline Valve implanted & $29 \mathrm{PM}$ & $25 \mathrm{PM}$ & $25 \mathrm{PM}$ & $29 \mathrm{PM}$ & $29 \mathrm{CM}$ & $29 \mathrm{HP}$ & $25 \mathrm{PM}$ & $29 \mathrm{PM}$ & $33 \mathrm{PM}$ \\
\hline Pulm. regurgitation & $S$ & $\mathrm{~S}$ & M-S & $S$ & $\mathrm{~S}$ & $\mathrm{~S}$ & $S$ & $S$ & $\mathrm{~S}$ \\
\hline Valve implanted & $23 \mathrm{PM}$ & $21 \mathrm{PM}$ & $23 \mathrm{PM}$ & $23 \mathrm{PM}$ & $27 \mathrm{CM}$ & $25 \mathrm{PM}$ & $23 \mathrm{PM}$ & $25 \mathrm{PM}$ & $27 \mathrm{PM}$ \\
\hline EUROscore & 4 & 7 & 3 & 5 & 5 & 4 & 5 & 3 & 5 \\
\hline Logistic EUROscore & 2.86 & 7.41 & 2.04 & 3.80 & 4.32 & 2.76 & 4.08 & 1.96 & 4.33 \\
\hline Left ventricle EF, \% & 50 & 40 & 55 & 55 & 50 & 55 & 55 & 55 & 45 \\
\hline Right ventricle & Sev.d Pres. & Mil.d Pres. & Mil.d Pres. & Mod.d Mod.im & Sev.d Pres. & Mil.d Mil.im & Sev.d Mil.im & Sev.d Sev.im & Mod.d Pres. \\
\hline ICU stay, d & 2 & 2 & 3 & 4 & 1 & 2 & 9 & 1 & 2 \\
\hline
\end{tabular}

M indicates male; F, female; M-S, moderate to severe; S, severe; Mil, mild; CM, carbomedics valve; PM, perimount magna valve; HM, Hancock porcine bioprosthesis; Mil.d, mildly dilated; Mod.d, moderately dilated; Sev.d: severely dilated; mil.im, mildly impaired; Mod.im, moderately impaired; Sev.im, severely impaired; Pres, preserved.

diagnosed with a small bowel carcinoid tumor with liver metastases having initially presented with abdominal pain, flushing, and dyspnoea (NYHA 3-4). Computed tomography $(\mathrm{CT})$ of the abdomen revealed multiple liver metastases and a small bowel mesenteric mass. A laparoscopic biopsy was consistent with a low-grade carcinoid tumor. All had good medium-term estimated prognoses but were deteriorating rapidly due to congestive heart failure.

All patients had clinical signs and symptoms of right heart failure and echocardiographic evidence of carcinoid heart disease, including thickened valves with regurgitation. Three patients also had left-sided heart failure. Right sided valves had severe involvement with severe tricuspid and pulmonary valve regurgitation except in 1 patient, when it was moderate. Examination of all patients revealed an elevated jugular venous pressure with prominent ' $\mathrm{V}$ ' waves and bilateral pedal oedema. The coronary arteries were free from disease on angiography except in one patient.
Because of the severity of cardiac symptoms, given that their estimated life expectancy was more than two years and based on the course of their carcinoid disease, the patients were accepted for surgery.

An intravenous infusion of Octreotide $(600 \mu \mathrm{g}$ in $60 \mathrm{~mL}$ $0.9 \%$ saline) was commenced a day before surgery at a rate of $5 \mathrm{~mL} / \mathrm{h}$, delivering $50 \mu \mathrm{g} / \mathrm{h}$ Octreotide. This continued during surgery and stopped 48 hours after the operation. Surgery was performed with the patient on aorto-bicaval cardiopulmonary bypass via a midline sternotomy. The patient's systemic temperature was lowered to $32^{\circ} \mathrm{C}$, with myocardial protection being achieved through antegrade cold blood cardioplegia, which was repeated every 30-45 minutes.

\section{RESULTS}

Patients' preoperative, intraoperative, and postoperative characteristics are shown in the Table. In patients with 
quadruple valve replacement, right-sided valves had more severe involvement then left-sided valves. Intraoperative echocardiography identified a PFO with right-to-left shunting in 1 patient (43\%) that was surgically confirmed and repaired. Notably, no defects were detected on preoperative transthoracic echocardiograms.

All patients had increased concentrations of urinary 5-hydroxyindoleacetic acid; levels were similar in patients with or without a PFO.

Patients included 5 women and 4 men. Mean preoperative NYHA was 3 (range 2-4), while postoperative NYHA was 1 in all patients. Mean age was 61 (range 55-70). Mean postoperative follow-up was 24 months (range 6-50 months, median 16 months). Mean additive EUROscore was 4.55 (range 3-7) and mean logistic EUROscore was 3.72 (range 1.96-7.41). All patients had good left ventricle, except one patient in whom it was mildly impaired. Right ventricle was severely dilated in 4 patients, moderately in 2, and mildly in 3 patients. Right ventricle was preserved in 5 patients, mildly impaired in 2 , moderately in 1 , and severely in 1 patient.

Right-sided valves were replaced with biological valves in 8 patients and with a mechanical valve in 1 patient. Left-sided valves were replaced with mechanical valve in 2 patients and with a biological valve in 1 patient. The valves used were Carbomedics bileaflet mechanical heart valves and CarpentierEdwards Perimount Magna Ease Heart Valve. One patient had tricuspid and pulmonary valve replacement and coronary artery bypass grafting. Carcinoid disease patients often have impaired liver function with higher risk of bleeding, so we prefer to use tissue valve on the right side. Moreover, if need arises, it is easier to place permanent pacemaker leads in the presence of tissue valves.

Right ventricular outflow tract reconstruction (pulmonary augmentation) with a pericardial patch was performed in all patients. Patients were transferred to the intensive care unit in a hemodynamically stable condition, with a modest dose of adrenaline and noradrenaline. They were temporarily paced for four days with subsequent recovery of their intrinsic sinus rhythm at which point the pacing was discontinued.

Mean ICU stay was 2.9 days (range 1-9 days, median 2 days) while mean hospital stay was 15 days (range 8-35 days, median 11 days). Mean chest drain was $547 \mathrm{~mL}$ (range 1002200, median $250 \mathrm{~mL}$ ). No patient experienced a carcinoid crisis. One patient was reopened for bleeding and there was one mortality (11\%). All excised valves had clinical and pathologic findings consistent with carcinoid heart disease.

One patient died of heart failure 9 days postoperatively in ICU. She was a 63-year-old female. She had normal LV, severely dilated RA and RV, mildly impaired RV with gross TR, and pulmonary stenosis. She underwent tricuspid and pulmonary valve replacement with tissue valve with single graft. Postoperatively her RV function became moderately to severely impaired. Her RV was unsuccessfully off-loaded with milrinone, nitric oxide, diuresis, and adrenaline. She also developed bilateral basal lung consolidation and died on day 10.

Functional improvement (eg, increased exercise tolerance, reduced lower extremity oedema, reduced ascites, resolution of orthopnoea, and paroxysmal nocturnal dyspnoea) was noted in all survivors. No thromboembolic events or bleeding complications occurred in our study population.

\section{DISCUSSION}

Bhattacharyya et al [Bhattacharyya 2011] noted that patients with carcinoid heart disease were referred for valve surgery if they had stable carcinoid tumor, severe valvular dysfunction, symptomatic, and had no other significant comorbidities.

The 5-year survival rate of patients without cardiac involvement ranges from $70 \%$ to $80 \%$ for all carcinoid tumors [Zuetenhorst 2003]. Surgical valve replacement rather than repair needs to be considered because of the severe retraction and fixation of the leaflets [Castillo 2008b] and it is the only effective treatment for carcinoid heart disease [Connolly 2002]. It is suggested that tricuspid and pulmonary valve replacement be considered when both valves are involved. It is true that many patients tolerate pulmonary regurgitation, but it is observed that patients who have a competent pulmonary valve have a much smoother postoperative recovery than do patients who have tricuspid valve replacement and pulmonary valvectomy. It appears that long-standing severe pulmonary valve regurgitation, as seen after pulmonary valvectomy, may have a detrimental effect on right ventricular remodelling [Connolly 2002]. In the pulmonary position, a stentless bioprosthesis is preferred because of its excellent hemodynamic characteristics and flexibility [Takahashi 2009].

There is some debate regarding selection of the prostheses in carcinoid patients. Mechanical prostheses are not ideal because patients with carcinoid syndrome often have extensive liver metastases and hepatic dysfunction, and they are at high risk of bleeding due to the use of lifelong anticoagulation. Early reports recommended using a mechanical prosthesis based on the assumption that circulating vasoactive tumor substances may damage bioprosthetic valves. Indeed, premature bioprosthetic degeneration has been reported in carcinoid patients [Ridker 1991].

Bioprosthetic valves can be potentially protected from the damaging effects of vasoactive substances by octreotide and hepatic artery interruption. There is less risk of involvement of bioprosthesis in the carcinoid process, once the primary carcinoid tumor is surgically removed [McDonald 1999].

The Mayo Clinic experience [Arghani 2010] suggested that cardiac valve replacement for symptomatic patients had a beneficial effect, causing a reduction in right ventricular size during the postoperative period. Komoda et al [Komoda 2011] suggested that the predictors of postoperative outcome may be not only signs of right ventricular failure, such as right ventricular volume overload and right ventricular function, but also preoperative left ventricular function in these patients. They also stressed the timing of cardiac surgery in patients with carcinoid heart disease. Presently, it is considered preferable for patients with stable carcinoid tumor to undergo surgery early after the onset of cardiac symptoms, as delay can result in worsening right ventricular failure and increase the risk of surgery. Hence, echocardiography is crucial in patients with carcinoid disease. 
Although most of our patients were severely ill, in NYHA class 3-4 and with symptoms of right heart failure, our results show an $89 \%$ early postoperative survival. In this study, we show significant improvement in functional capacity after valve replacement for carcinoid heart disease, without valverelated complications and low perioperative mortality.

\section{Conclusion}

Carcinoid heart disease is an uncommon and complex form of valvular heart disease. Cardiac involvement is a major cause of morbidity and mortality. Despite metastatic disease that limits longevity, valve replacement in symptomatic patients is a reasonable option with survival benefit, low early-postoperative mortality, without valve-related complications and with functional improvement. Cardiac assessment is required in all patients with carcinoid disease from the earliest time of medical treatment to improve patients' results.

\section{REFERENCES}

Arghani A, Connolly HM, Abel MD, Schaff HV. 2010. Quadruple valve replacement in patients with carcinoid heart disease. J Thoracic Cardiovasc Surg 140:1432-4.

Bernheim AM, Connolly HM, Hobday TJ, Abel MD, Pellikka PA. 2007. Carcinoid heart disease. Prog Carciovasc Dis 49:439-51.

Bhattacharyya S, Raja SG, Toumpanakis C, Caplin ME, Dreyfus GD, Davar J. 2011. Outcomes, risks and complications of cardiac surgery for carcinoid heart disease. Eur J Cardiothorac Surg 40:168-72.

Castillo JG, Filsoufi F, Rahmanian PB, et al. 2008. Early and late results of valvular surgery for carcinoid heart disease. J Am Coll Cardiol 51:1507-9.

Castillo JG, Filsoufi F, Rahmanian PB, et al. 2008. Early and late results of valvular surgery for carcinoid heart disease. J Am Coll Cardiol 51:1507-9.

Connolly HM. 2001. Carcinoid heart disease: medical and surgical considerations. Cancer control 8:454-60.

Connolly HM, Schaff HV, Mullany CJ, Rubin J, Abel MD, Pellikka PA.
2001. Surgical management of left-sided carcinoid heart disease. Circulation 104:12 (Suppl. 1):I36-I40

Connolly HM, Schaff HV, Mullany CJ, Abel MD, Pellikka PA. 2002. Carcinoid heart disease: impact of pulmonary valve replacement in right ventricular function and remodeling. Circulation 106(suppl):I51-6.

Ferrans V, Roberts W. 1976. The carcinoid endocardial plaque: an ultrastructural study. Hum Pathol 7:387-409.

Gustafsson BI, Hauso O, Drozdov I, Kidd M, Modlin IM. 2008. Carcinoid heart disease. Int J Cardiol 129:318-24.

Komoda S, Komoda T, Pavel ME, et al. 2011. Cardiac surgery for carcinoid heart disease in 12 cases. Gen Thorac Cardiovasc Surg 59:780-5.

McDonald ML, Nagorney DM, Connolly HM, Nishimura RA, Schaff HV. 1999. Carcinoid heart disease and carcinoid syndrome: successful surgical treatment. Ann Thorac Surg 67:537-9.

Moertel C. Karnofsky memorial lecture. 1987. An odyssey in the land of small tumors. J Clin Oncol 5:1502-22.

Pellikka P, Tajik A, Khandheria B, et al. 1993. Carcinoid heart disease: clinical and echocardiographic spectrum in 74 patients. Circulation 87:1188-96.

Raja SG, Bhattacharyya S, Davar J, Dreyfus GD. 2010. Surgery for carcinoid heart disease: current outcomes, concerns and controversies. Future Cardiol 6:647-55

Ridker PM, Chertow GM, Karlson EW, Neish AS, Schoen FJ. 1991. Bioprosthetic tricuspid valve stenosis associated with extensive plaque deposition in carcinoid heart disease. Am Heart J 121:1835-8.

Roberts W. 1997. A unique heart disease associated with a unique cancer: carcinoid heart disease. Am J Cardiol 80:251-6.

Takahashi H, Okada K, Asano M, Matsumori M, Morimoto Y, Okita Y. 2009. Bioprosthetic pulmonary and tricuspid valve replacement in carcinoid heart disease from ovarian primary cancer. Circ J 73:1554-6.

Zuetenhorst JM, Bonfrer JM, Korse CM, Bakker R, Tinteren H, Taal BG. 2003. Carcinoid heart disease: the role of urinary 5 -hydroxyindoleacetic acid excretion and plasma levels of atrial natriuretic peptide, transforming growth factor-beta and fibroblast growth factor. Cancer 97:1609-15. 